\title{
Delayed alternation in rats with position preferences"
}

\author{
R. O'CONNELL \\ San Fernando Valley State College, Northridge, Calif. 91324
}

Spontaneous alternation of rats with relatively strong and weak position preferences was examined with intertrial intervals of 1,30 , and $60 \mathrm{~min}$. Differences were not found between the preference groups in frequency of alternation at different ITIs. Strong-preference Ss had shorter Trial 1 choice latencies and weak-preference Ss were more likely on Trial 2 to make an initial body entry of the previously chosen arm before alternating. Alternation was sometimes lowest with 30 -min ITI.

The tendency of the rat to spontaneously alternate (SA) in its entry of the arms of a T maze has generated a succession of explanations (O'Connell, 1965a). Initially, there was a reluctance to interpret the $\mathrm{SA}$ displayed on a second trial as a result of learning occurring on the first trial because most learning requires more than one trial and because of the apparently quite temporary aftereffect of an initial trial (see Dember, 1961, p. 230). Rarity of one-trial learning was never much of an argument and is less so today. Data indicating the persistence of SA over long intertrial in tervals (ITI) continue to accumulate (Denny \& Leckart, 1965; O'Connell, $1965 \mathrm{~b}$; Squire 1969) and support attempts to interpret SA using a learning model (O'Connell, 1965a) rather than the earlier stimulus satiation model (Glanzer, 1953).

Thompson (1959) trained rats on the repeated reversal of a position habit in a $T$ maze and found that Ss with a strong position preference, when trained to their nonpreferred side, showed retention $1 \mathrm{~min}$ after training but not after 1 or $5 \mathrm{~h}$. The fact that the rewarded response was more likely to be repeated after a short ITI is evidence against one interpretation of $\mathrm{SA}$ and the consolidation process (see O'Connell, $1965 \mathrm{a}$ ). The work reported here was an attempt to determine if results analogous to Thompson's could be found with SA: Do rats with strong position preferences display a greater decrease in SA than Ss with weak preferences as ITI is lengthened?

\section{STUDY 1 \\ Method}

Forty Sprague-Dawley albino rats, obtained from Dr. Roberts' colony at UCLA, were maintained ad lib and ranged in age from 120 to 150 days when the study began. In the $\mathrm{T}$ maze

\footnotetext{
* A part of this research was supported by NSF funds through the Committee on Research, UCLA. David Stallard, Douglas Sue, and Henry Weber assisted in data collection.
}

used in this study, Ss had previously received 5 to 10 daily position-preference test trials and had been tested under eight stimulus-change conditions, using a procedure similar to one previously described (O'Connell, 1964). On a given position-preference test day, both maze arms, the endboxes, and the choice area had walls and floor lined with posterboard of the same brightness. From day to day the liners varied among four brightnesses: white, light gray, dark gray, and black.

From the 40 Ss, 18 "strong-preference" rats were selected which, with two exceptions, had selected the same side at least seven of eight times during stimulus-change tests, or selected the same side on at least $80 \%$ of the position-preference tests. Tallying all responses from both tests, the preferred side, which for all but two Ss was the right side, was chosen $80.4 \%$ of the time. Eighteen "weak-preference" Ss were selected such that, with one exception, all met neither of the criteria for inclusion in the strong-preference group; overall choice of each of these Ss' most frequently chosen side was $59.9 \%$.

The $\mathrm{T}$ maze, described in detail elsewhere (O'Connell, 1964), had added a $4 \frac{1}{2} \times 9$ in. startbox, painted flat gray, with a wooden swing-lift outer door and a glass guillotine door separating it from the choice area. Guillotine doors about midway down the choice arms prevented retracing. The walls and floors of the choice area, arms, and endboxes were uniformly covered each day with a light gray, dark gray, or black posterboard. Each brigh tness was used two different days, and liners were changed each day in an attempt to maintain the visual novelty of the maze. The maze was surrounded with white sheeting.

Each $S$ received one alternation test, consisting of two trials, on each of 6 days. ITIs of 1,30 , and $60 \mathrm{~min}$ were used. During this interval, $S$ was confined in his living cage in the experimental room. Ss were run in groups of six, with one of the six possible orders of the three ITIs administered to each rat during its first 3 experimental days and reversed for the last 3 days. On a given day, all $S$ s in the group(s) being tested which were to receive the same ITI were tested successively and followed by the two sets of Ss which were to receive each of the other ITIs. The order of ITIs differed for each of the experimental days.

On each trial, after $S$ had passed a guillotine door in an arm, it was allowed to remain in the goalbox area for $1 \mathrm{~min}$ before being removed to its living cage. After each trial the plastic floor covers were wiped with tissues wetted with Wizard Evergreen room deodorizer spray. Removal of scent-trail cues was intended to prevent these from being confounded with ITI, since, for the longer ITIs, other rats were given a trial between a rat's first and second trials.

Results and Discussion

The strong-preference Ss displayed $67 \%, 53 \%$, and $69 \%$ SA with ITIs of 1 , 30 , and $60 \mathrm{~min}$, respectively. For weak-preference Ss the corresponding values were $72 \%, 53 \%$, and $72 \%$. The chance level of SA was calculated for each $S$ by counting the number of occasions on which his first trial on one day differed from his first trial the following day (see Douglas \& Isaacson, 1965). The first trial on the sixth day was compared with the first trial on the first day to make a total of six paired first trials. The chance level was $46 \%$ for strong-preference Ss and $44 \%$ for those with weak preference.

Each S's chance percentage of SA was subtracted from his percentage of SA for a given ITI, and the differences evaluated with the sign test. For both preference groups these differences were significant at at least the .05 level for 1- and 60-min ITIs. The two preference groups did not significantly differ in SA for any ITT. It is difficult to either believe or explain the function unexpectedly obtained between SA and ITT If real, it would be analogous to the "Kamin effect" and invite similar explanatory approaches (see Klein \& Spear, 1970).

For a direct comparison of ITIs, the number of SAs during the two tests with a given ITI were pooled for the three Ss within each preference group which received ITIs in the same sequence. With six sequences in each group, this resulted in a 12 by 3 table with one column for each ITI. In each row the frequencies were ranked and a Friedman two-way ANOVA by ranks (with three-way ties eliminated) was used to test column differences (Siegel, 1956). The lower frequency of $\mathrm{SA}$ in the $30-\mathrm{min}$ ITI condition 
produced a significant column effect $\left(x^{2}=8.55, \mathrm{df}=2, \mathrm{p}<.02\right)$.

For $\mathrm{Ss}$ in the strong-preference group, a tally was made of the frequency with which first-trial choices were to what had been judged previously to be each $S$ 's preferred side. Only $53.7 \%$ of the choices were to that side. To determine more sensitively if evidence existed for preference differences between the groups, the numbers of alternations were compared for subgroups from strong- and weak-preference groups which were matched on the number of first trials to one side. Only five Ss had just three first-trial choices to one side; many had four to one side and these subgroups did not differ in SA. Among Ss with five first-trial choices to one side, Ss from the weak-preference group had an average of twice as many SAs $(t=2.45, \quad d f=8 . p<.05)$. This provides weak evidence of preference differences; however, such differences were clearly much less pronounced at the time of testing than one would desire. In part, this may be attributable to the fact that preference sorting was done on the basis of responses made up to a month in advance of SA testing. The second study was executed to determine the effect of preferences on SA when preference is assessed at the time of $\mathrm{SA}$ testing.

\section{STUDY 2}

\section{Method}

Nine groups, each consisting of six male Sprague-Dawley albino rats, were used Ss were between 90 and 120 days of age at the start of the study and were individually housed and maintained ad lib throughout. Five groups were obtained from Roberts' colony and had previously had four 3-min open-field exposures. Four groups were obtained from Holtzman and had been tested under eight stimulus-change conditions. All Ss were given a week of daily table-top handling followed by one position-preference test in the $T$ maze on each of 4 days. Arms were of uniform brightness on any day; a different brightness was used each day. Preference tests were immediately followed by 6 days of SA testing with the three ITIs. The maze and procedure were the same as in Study 1.

\section{Results}

Position preference was determined on the basis of each S's responses over the four preference trials and the six first trials of SA days. Assuming no position preferences, $89 \%$ of the Ss would be expected to make 5,6 , or 7 choices to the same side and $11 \%$ would be expected to make 8,9 , or 10 . In fact, $61 \%$ selected the same side 8 or more times $(p<.001)$, which indicates the presence of position preferences.

After testing the 54 Ss, 18 strong-preference $S$ s wele chosen which had $10(\mathrm{~N}=2), 9(\mathrm{~N}=6)$, or 8 $(\mathrm{N}=10)$ responses to one side, and 18 weak-preference $\mathrm{Ss}$ which had 5 $(\mathrm{N}=5), 6(\mathrm{~N}=10)$, or $7 \quad(\mathrm{~N}=3)$ responses to one side. Selection was made so that three $S s$ in each of the preference groups had received alternation tests in one of the six orders of ITIs. The sequences in which ITIs were administered within a day were also well balanced in each group.

The strong-preference Ss displayed $64 \%, 47 \%$, and $42 \% \mathrm{SA}$ with ITIs of 1 , 30 , and $60 \mathrm{~min}$, respectively. For weak-preference Ss the corresponding values were $78 \%, 56 \%$, and $58 \%$. Chance SA was not computed from first-trial alternation since these data had been used to assign Ss to groups and could be expected to underestimate chance $\mathrm{SA}$ for strong-preference Ss. If chance SA of $50 \%$ is assumed, the obtained distribution of frequencies of two, one, or no SAs differs from that expected only for weak-preference Ss with 1-min ITI $\left(x^{2}=11.3, \quad d f=2\right.$, $p<.01)$. For none of the ITIs does the frequency of alternation of weak-preference Ss significantly exceed that of strong-preference Ss.

The difference between ITIs was again tested with the Friedman test and found significant $\left(x^{2}=9.12\right.$, $\mathrm{df}=2, \quad \mathrm{p}<.02)$. An ANOVA for repeated measures performed on these same grouped data also showed a significant ITI effect ( $F=9.33$, $\mathrm{df}=2 / 10, \quad \mathrm{p}<.05), \quad$ with neither preference nor Preference by ITI interaction significant. With one exception, the relation between $\mathrm{SA}$ and ITI on the first and last 3 days was similar to that overall: for the first 3 days weak-preference Ss had $61 \%$ SA for 1- and 60-min ITI and $28 \% \mathrm{SA}$ for $30 \min (p<.05)$.

Full body entry of an arm which did not take $S$ beyond a guillotine door was also recorded. When choice on Trial 2 was defined as the first arm entered in this manner, $64 \%$ of the changes produced in the data were subtractions rather than additions of a SA $(\mathrm{p}<.01)$. That is, if Ss first made a body entry of one arm on Trial 2 and then entered the other arm past the door, the first entry was more likely to be to the side chosen on Trial 1. The reduction in SA by the body-entry measure was more pronounced with the elimination of questionable "pseudoalternations": cases in which on both trials $S$ had a body entry to the left (right) followed by final choice of the right (left) arm. With nine pseudoalternations removed, for strong-preference Ss SA by the body-entry criterion on Trial 2 was $61 \%, 40 \%$, and $10 \%$ for $1-30$, and 60-min ITIs, respectively. For weak-preference $S$ s the corresponding percentages were $49 \%, 45 \%$, and $60 \%$; for the 1-min ITI this drop was significant $(p<.05)$ and differed from that of strong-preference $\mathrm{Ss}(\mathrm{p}<.05)$.

Choice latencies, measured from the lifting of the startbox door until $S$ passed an arm door, did not appear to meet the assumptions necessary for an ANOVA, so the data were analyzed nonparametrically. Each preference group was reduced to its six constituent subgroups of three Ss each. For each ITI within each trial the latencies were summed over the two tests for each $S$ and over the three Ss. Of the 36 latency values thus obtained for Trial 1, $83 \%$ were greater than the corresponding values for Trial $2(p<.01)$. Eighteen comparisons between high- and low-preference subgroups on Trial 1 revealed high-preference Ss had shorter latencies (Wilcoxon $\mathrm{T}=39, \mathrm{n}=18$, $\mathrm{p}<.05)$. This difference between preference groups was in the same direction on Trial 2 but failed to reach significance. On Trial 2 latencies generally increased with ITI, with rather similar values for 1 - and $30-\mathrm{min}$ ITIs; the Wilcoxon test between 1- and 60-min ITIs did not reach signifiance. DISCUSSION

The latency difference between the preference groups on Trial 1 and the direction of the SA differences between the groups suggest that the sorting of Ss into groups was somewhat more successfully achieved in the second study. However, there was no firm support for the hypothesis of group differences in the relation between SA and ITI. With Trial 2 choice defined as the last arm entered, SA was highest for the shortest ITI and appeared about the same for $30-$ and $60-\mathrm{min}$ ITIs. The trend of the latency scores suggests similar times for the shorter ITIs and a longer latency with $60-\mathrm{min}$ ITI.

The reappearance of the $U$ function between SA and ITI indicates that such a relation may be real under some conditions. The best guess would be that these conditions include the absence of strong position preference and the presence of anxiety. About half the Ss in Study 2 urinated in the maze at least once and, from the first 3 days to the last 3 , the percentage of SA for each ITI increased on the average $22 \%$ for weak-preference Ss and $17 \%$ for strong-preference Ss.

The significantly lower level of SA found with the body-entry measure was much more pronounced in weak-preference Ss with a short ITI. Weak-preference Ss frequently started Trial 2 with a body entry of their 
Trial 1 choice and then alternated. Strong-preference Ss were not likely to leave the arm chosen on Trial 1 if they reentered it on Trial 2. Hughes (1966), investigating the effect of stimulus change on SA, found it did affect frequency of partial body entries but not the arm which was finally selected. The present study indicates that position preference also may be reflected more strongly in variations in body entry of the nonselected side than in frequencies of final choice.

The primary hypothesis gains support from Iwahara's (1960) finding that shocked rats, with strong position preferences, showed an unusually sharp decline in SA as ITI was increased from $0 \mathrm{sec}$ to $3 \mathrm{~min}$. In further examination of the hypothesis, position preference differences could be obtained by appropriate selection of the strains used (see Douglas \& Isaacson, 1965), with the confounding this would entail, or preferences could be artificially created by using arms of different brightness (see Eisenberg, Myers, Sanders, \& Shanab, 1970; O'Connell, 1964; Pate \& DeLoache, 1970). Preferences could also be established by variations in amount of reward training. SA data obtained under these conditions would invite comparisons with results of studies of proactive inhibition (e.g., Gleitman \& Jung, 1963).

\section{REFERENCES}

DEMBE R, W. N. Alternation behaviox. In $D$. W. Fiske and S. R. Maddi (Eds.), Functions of varied experience. Homewood, Ill: Dorsey Press, 1961. Pp. 227-252.

DENNY, M. R., \& LECKART, B Alternation behavior: Learning and Comparative \& Physiological Psychology, $1965,60,229-232$. extinction one trial per day. Journal of

DOUGLAS, R., \& ISAACSON, R. Homogeneity of single trial response tendencies and spontaneous alternation in the 'T-maze. Psychological Reports, 1965 , 16. 87-92.

EISENBERGER, R., MYERS, A. K., SANDERS, R., \& SHANAB, M. E Stimulus control of spontaneous alternation. Journal of Comparative \& Physiological Psychology, 1970, 70, 136-140.

GLANZER, M. Stimulus satiation: An explanation of spontaneous alternation and related phenomena. Psychological Review, 1953,60, 257-268.

GLEITMAN, H., \& JUNG, L. Retention in rats: The effect of proactive interference. Science, 1963, 142,1683-1684.

HUGHES, R. N. Stimulus-change effects of forcing in alternation behavior. Psychological Reports, 1966, 19, 515-518.

IWAHARA, S. Effect of intertrial interval upon alternation behavior in the shocked rat. Psychological Reports, 1960, 6, 266.

KLEIN, S. B., \& SPEAR, N. E. Forgetting by the rat after intermediate intervals ("Kamin effect") as retrieval failure. Journal of Comparative \& Physiological Psychology, 1970, 71, 165-170.

O'CONNELL, R. H. Comparison of altemation and response to stimulus change. Journal of Comparative \& Physiological Psychology, 1964, 57, 362-366.

O'CONNELL, R. H. Trials with tedium and titillation. Psychological Bulletin, 1965a, $63,170-179$.

O'CONNELL, R. H, Spontaneous alternation following long intertrial intervals. Psychological Reports, $1965 \mathrm{~b}$, 17, 131-134.

PATE, J. L., \& DeLOACHE, J. Brightness and direction as cues for spontaneous alternation behavior. Psychonomic Science, 1970, 18, 27-28.

SIEGEL, S. Nonparametric statistics for the behavioral sciences, New York: McGraw-Hill, 1956.

SQUIRE, L. R. Effects of pretrial and posttrial administration of cholinergic and anticholinergic drugs on spontaneous alternation. Journal of Comparative \& Physiological Psychology, 1969, 69, 69-75.

THOMPSON, R. Transient memory in albino rats. Science, $1959,129,842-843$.

\section{Temporal effects of RNase and DNase in disrupting acquired escape behavior in regenerated planaria}

\author{
DANIEL $J$. LACEY \\ Department of Anatomy-Physiology \\ Indiana University, Bloomington, Ind. 47401
}

Trained planaria, transected immediately after the last training trial and regenerated in RNase, exhibited impairment of an acquired escape pattern of responses. Regeneration in DNase resulted in no response decrement. Ss transected $24 \mathrm{~h}$ after completion of the last training sessions and regenerated in either RNase or DNase, displayed significant response decrement Results suggest a time-dependent involvement of RNA and DNA in maintenance of the acquired response pattern.

The possible roles of RNA and DNA in learning and memory have received much attention by investigators in the past decade. 1 Corning \& John (1961) trained planaria to make a contraction to a light stimulus in order to avoid an electric shock. Ss were then transected and allowed to regenerate, half in pond water, the other half in pond water containing RNase. The regenerated animals were tested for 3 consecutive days for retention of the conditioned response. Significant results were obtained when regenerated-head planaria, kept in RNase, were tested. This group behaved randomly as though it had not been conditioned. It was assumed that memory was impaired and that RNA was involved in retention of a conditioned response. All other regenerated Ss evidenced no deficit in performance of the response.

Experiments like that of Corning and John, purporting to demonstrate conditioning in planaria and other species, have been criticized on many grounds. Sensitization rather than conditioning of the planaria seems to have been indicated in these experiments. In addition, the data on whether or not planaria are even capable of learning are extremely equivocal (Jensen, 1965). However, the above study is illustrative of those favoring the implication of RNA in learning and memory. The role of DNA has been less extensively studied, perhaps because of its lesser lability than RNA. A nucleoprotein, highly resistant to alternation, could not transcribe the changes necessary for learning and retention. However, it would seem impossible for a learned response to be maintained unless DNA were involved, for after the initial altered RNA is depleted, from where would come the instructions to keep encoding this newly formed structure? This suggests that some structural modification must occur to DNA so that it could keep transcribing the altered RNA which would, by some complex and uncertain process, enable the response to be retained over a period of time.

The purpose of the present experiment was to determine if there was a time-dependent involvement of DNA and RNA in the maintenance of an acquired response pattern. Planaria were taught to crawl to a particular position in a glass bowl to escape an aversive light stimulus. By transecting the animals at different times after training and placing them in solutions containing RNase or DNase to regenerate, it could be ascertained whether RNA or DNA were involved in retention and if there was a temporal relationship. Testing for retention of responses after regeneration enables testing of the hypothesis that altered RNA must migrate back to the nucleus of the cell and bring about structural changes in DNA. The implication is that if the migration if interrupted along the way, for example by electroshock or more probably by enzymatic activity,

memory consolidation would be 